\title{
Double and negative reflection of cold atoms in non-Abelian gauge potentials
}

\author{
Gediminas Juzeliūnas, ${ }^{1,2}$ Julius Ruseckas, ${ }^{1}$ Andreas Jacob, ${ }^{3}$ Luis Santos, ${ }^{3}$ and Patrik Öhberg ${ }^{4}$ \\ ${ }^{1}$ Institute of Theoretical Physics and Astronomy of Vilnius University, A. Goštauto 12, Vilnius 01108, Lithuania \\ ${ }^{2}$ Department of Physics and Technology, Vilnius Pedagogical University, Studentu 39, Vilnius 08106, Lithuania \\ ${ }^{3}$ Institut für Theoretische Physik, Leibniz Universität, Hannover D3016\%, Germany \\ ${ }^{4}$ SUPA, School of Engineering and Physical Sciences, \\ Heriot-Watt University, Edinburgh EH14 4AS, United Kingdom
}

(Dated: November 21, 2018)

\begin{abstract}
Atom reflection is studied in the presence of a non-Abelian vector potential proportional to a spin- $1 / 2$ operator. The potential is produced by a relatively simple laser configuration for atoms with a tripod level scheme. We show that the atomic motion is described by two different dispersion branches with positive or negative chirality. As a consequence, atom reflection shows unusual features, since an incident wave may split into two reflected ones at a barrier, an ordinary specular reflection, and an additional non-specular one. Remarkably, the latter wave can exhibit negative reflection and may become evanescent if the angle of incidence exceeds a critical value. These reflection properties are crucial for future designs in non-Abelian atom optics.
\end{abstract}

PACS numbers: 03.75.-b, 42.50.Gy, 42.25.Bs

Introduction Atomic mirrors, created by optical [1] or magnetic [2] potential barriers, play a crucial role in atom optics enabling to manipulate matter waves. Wave reflection at a mirror is typically specular, where the reflection angle equals the incidence one. However, richer reflection scenarios are also possible. For optical waves a double reflection appears in optically active media, such as in chiral liquids characterized by different refractive indices for left and right polarized light [3]. This manifests itself as a tiny splitting of the reflected wave into two parts [4]. An additional striking example is Andreev reflection [5, 6] in which an electron incident at the interface between a normal metal and a superconductor is reflected to a positively charged hole propagating backwards, where the missing charge of $2 e$ enters the superconductor as a Cooper pair.

Artificial electromagnetism for cold neutral atoms is attracting a growing attention. A proper manipulation of atoms in optical lattices may allow for the observation of Hofstadter butterfly energy spectra [7] and paradoxical geometries [8], as well as for the generation of nonAbelian gauge potentials [9]. Alternatively, Abelian and non-Abelian gauge potentials may be induced by means of laser fields acting on atoms in, respectively, lambda [10] and tripod [11, 12, 13] schemes of electronic levels. Non-Abelian gauge potentials lead to a number of distinctive properties, e.g. modification of the metal-insulator transition 14] or non-Abelian Aharanov-Bohm interferometric effects [9, 15]. Recently, it was shown 16] that a tripod-scheme provides quasi-relativistic physics for cold atoms, similar to that for electrons in graphene [17].

In this Letter we analyze atom reflection in the presence of a non-Abelian vector potential proportional to a spin- $1 / 2$ operator produced by a relatively simple laser arrangement for tripod-scheme atoms. We show that the appearance of two different dispersion branches with pos-
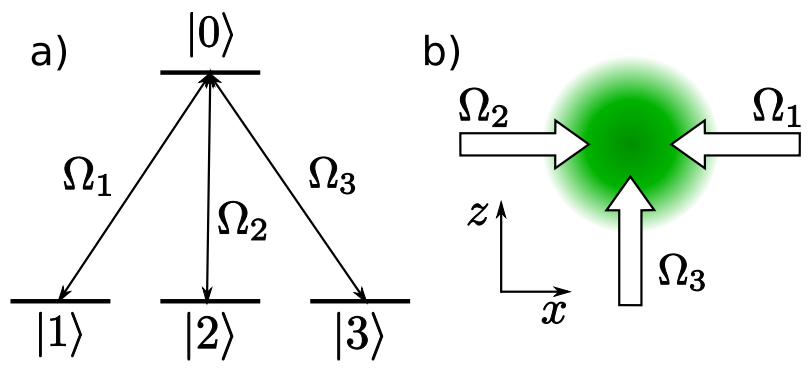

FIG. 1: (Color online) Three light fields acting on atom in a tripod configuration of energy levels involved.

itive or negative chirality leads to a double reflection at the mirror, an ordinary specular reflection, and an additional non-specular one. Remarkably, the latter can exhibit a negative reflection, resembling the Andreev reflection [5, 6]. The negatively reflected wave becomes evanescent if the angle of incidence exceeds a critical value. These reflection properties could become crucial for the design of future non-Abelian atom optics devices, as e.g. non-Abelian atom interferometers [9, 15].

Adiabatic motion of tripod atoms In the following we consider an atom with a tripod electronic level scheme $\{|0\rangle,|1\rangle,|2\rangle,|3\rangle\}$ (see Fig. 11 $a$ ) under the influence of three stationary laser beams [11, 16, 18, 19]. The $j$-th laser induces a resonant atomic transition (with a Rabi frequency $\Omega_{j}$ ) between $|j\rangle$ and $|0\rangle$. These can be for instance the transition $2^{3} S_{1} \leftrightarrow 2{ }^{3} P_{0}$ in ${ }^{4} \mathrm{He}^{*}$ or the transition $5 S_{1 / 2}(F=1) \leftrightarrow 5 P_{3 / 2}(F=0)$ in ${ }^{87} \mathrm{Rb}$.

The electronic Hamiltonian of the tripod system reads in the interaction representation [1]

$$
\hat{H}_{0}=-\hbar|0\rangle\left(\Omega_{1}\langle 1|+\Omega_{2}\langle 2|+\Omega_{3}\langle 3|\right)+\text { H.c. } .
$$

Thus $|0\rangle$ is coupled only to the bright state $|B\rangle=\left(\Omega_{1}^{*}|1\rangle+\right.$ $\left.\Omega_{2}^{*}|2\rangle+\Omega_{3}^{*}|3\rangle\right) / \Omega$, where $\Omega=\left(\left|\Omega_{1}\right|^{2}+\left|\Omega_{2}\right|^{2}+\left|\Omega_{3}\right|^{2}\right)^{1 / 2}$ 
is the total Rabi frequency. The two states $|B\rangle$ and $|0\rangle$ split into a dressed doublet $| \pm\rangle=(|B\rangle \pm|0\rangle) / \sqrt{2}$ with energies $\pm \hbar \Omega$. The remaining two eigenstates $\left|D_{j}\right\rangle(j=$ $1,2)$, known as dark states, are orthogonal to $|B\rangle$ and hence are decoupled from the light fields, $\hat{H}_{0}\left|D_{j}\right\rangle=0$. We assume that the light fields are sufficiently strong, so that $\Omega$ is large compared to the two-photon detuning due to the laser mismatch and/or Doppler shift. The dark states are thus well separated in energies from the doublet $| \pm\rangle$, and the internal atomic state evolves within the dark state manifold. The full atomic state-vector $|\Phi\rangle$ can then be expanded in terms of the dark states, $|\Phi\rangle=$ $\sum_{j=1}^{2} \Psi_{j}(\mathbf{r})\left|D_{j}(\mathbf{r})\right\rangle$, where $\Psi_{j}(\mathbf{r})$ is a wave-function for the center of mass motion of an atom in the $j$-th dark state. The two-component spinor-like wavefunction $\Psi=$ $\left\{\Psi_{1}, \Psi_{2}\right\}^{T}$ obeys the Schrödinger equation $i \hbar \partial \Psi / \partial t=$ $H \Psi$, with the center of mass Hamiltonian [1]

$$
H=\frac{1}{2 M}(-i \hbar \nabla-\mathbf{A})^{2}+V+\Phi,
$$

where $M$ is the atomic mass. The gauge potentials A and $\Phi$ emerge due to the spatial dependence of the dark states. The $2 \times 2$ matrix $\mathbf{A}$ with the elements $\mathbf{A}_{n m}=i \hbar\left\langle D_{n}(\mathbf{r}) \mid \nabla D_{m}(\mathbf{r})\right\rangle$ represents the effective vector potential known as the Mead-Berry connection 11, 16, 19, 20, 21, 22]. The $2 \times 2$ matrix $\Phi$ with elements $\Phi_{n m}=\left(\hbar^{2} / 2 M\right)\left\langle D_{n}(\mathbf{r}) \mid \nabla B(\mathbf{r})\right\rangle\left\langle B(\mathbf{r}) \mid \nabla D_{m}(\mathbf{r})\right\rangle$ acts as an effective scalar potential. Finally, the $2 \times 2$ matrix $V$ with elements $V_{n m}=\left\langle D_{n}(\mathbf{r})|\hat{V}| D_{m}(\mathbf{r})\right\rangle$ is an external potential for the dark-state atoms. Here $\hat{V}=\sum_{j=1}^{3} V_{j}(\mathbf{r})|j\rangle\langle j|$, with $V_{j}(\mathbf{r})$ being the trapping potential for an atom in the $j$-the internal state. Note that the potential $V_{j}$ can also accommodate a detuning of the $j$-th laser from the resonance of the $j \rightarrow 0$ transition.

Laser arrangement Although elaborate laser configurations may allow for a wealth of possible gauge potentials in the tripod scheme [11], here we concentrate on a relatively simple laser setup providing non Abelian potentials. The first two laser beams are assumed to counterpropagate with the same intensity along the $x$ axis, $\Omega_{1}=\Omega \sin \theta \mathrm{e}^{-i \kappa_{0} x} / \sqrt{2}$ and $\Omega_{2}=\Omega \sin \theta \mathrm{e}^{i \kappa_{0} x} / \sqrt{2}$, and the third one propagating in the $z$-direction [16], $\Omega_{3}=\Omega \cos \theta \mathrm{e}^{i \kappa_{0} z}$. Here $\kappa_{0}$ is the wave-number, and the mixing angle $\theta$ characterizes the relative intensity of the third laser. A set of two dark states is then given by

$$
\begin{aligned}
& \left|D_{1}\right\rangle=2^{-1 / 2}(|\tilde{1}\rangle-|\tilde{2}\rangle) e^{-i \kappa^{\prime} z}, \\
& \left|D_{2}\right\rangle=\left[2^{-1 / 2} \cos \theta(|\tilde{1}\rangle+|\tilde{2}\rangle)-\sin \theta|3\rangle\right] e^{-i \kappa^{\prime} z},
\end{aligned}
$$

with $\kappa^{\prime}=\kappa_{0}(1-\cos \theta)$, where the modified atomic state-vectors $|\tilde{1}\rangle=|1\rangle \exp \left[i \kappa_{0}(x+z)\right]$ and $|\tilde{2}\rangle=$ $|2\rangle \exp \left[-i \kappa_{0}(x-z)\right]$ accommodate the phases of the laser fields. An additional phase factor $\exp \left(i \kappa^{\prime} z\right)$ introduces a shift in the origin of the momentum $\mathbf{k} \rightarrow \mathbf{k}+\kappa^{\prime} \mathbf{e}_{z}$. By imposing $\cos \theta=\sqrt{2}-1$, the vector potential becomes

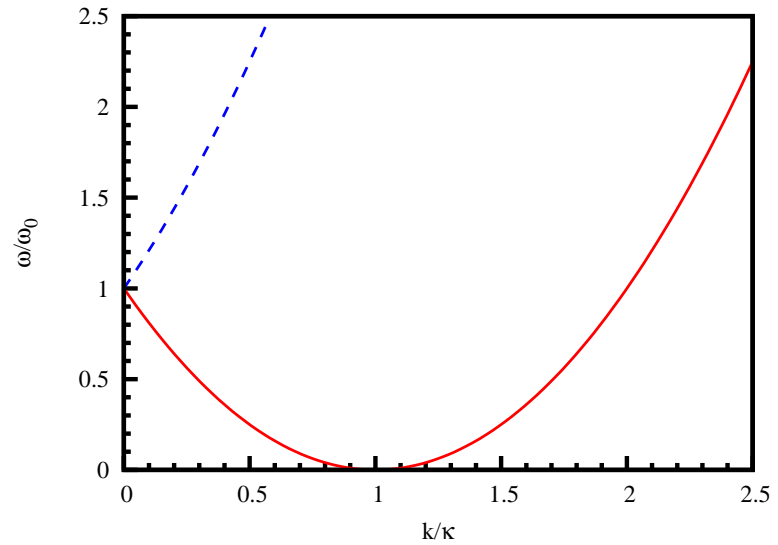

FIG. 2: (Color online) Upper (blue dashed) and lower (red solid) dispersion branch for tripod atoms in light fields.

$\mathbf{A}=-\hbar \kappa \sigma_{\perp}$, where $\sigma_{\perp}=\mathbf{e}_{x} \sigma_{x}+\mathbf{e}_{z} \sigma_{z}$ is the operator of spin $1 / 2$ in the $x z$ plane, and $\kappa=\kappa_{0} \cos \theta \approx 0.414 \kappa_{0}$. The Cartesian components $A_{x}$ and $A_{z}$ are proportional to the Pauli matrices $\sigma_{x}$ and $\sigma_{z}$ which do not commute, so the vector potential $\mathbf{A}$ is non-Abelian. Note that similar non-Abelian gauge potentials can also be induced by means of standing waves in a tripod setup 12, 13] or by state selective tunneling in optical lattices [9].

Furthermore we take the trapping potentials $V_{1}=V_{2}$ and $V_{3}-V_{1}=\hbar \kappa_{0}^{2} \sin ^{2} \theta / 2 M$. This can be achieved by detuning properly the third laser from the two-photon resonance. Hence the overall trapping potential $V+\Phi$ becomes proportional to the unit matrix, both dark states being affected by the same potential $V_{1} \equiv V_{1}(\mathbf{r})$, giving

$$
H=\frac{1}{2 M}\left(-i \hbar \nabla+\hbar \kappa \sigma_{\perp}\right)^{2}+V_{1}(\mathbf{r}) .
$$

Dispersion law We shall consider a two dimensional case where the atomic motion is confined to the $x z$ plane. If the trapping potential $V_{1}$ is constant, the eigenfunctions of the Hamiltonian (5) are the plane waves

$$
\Psi_{\mathbf{k}}^{ \pm}(\mathbf{r})=g_{\mathbf{k}}^{ \pm} e^{i \mathbf{k} \cdot \mathbf{r}}, \quad g_{\mathbf{k}}^{ \pm}=\frac{1}{2}\left(\begin{array}{c}
1 \mp i e^{i \varphi_{\mathbf{k}}} \\
-i \pm e^{i \varphi_{\mathbf{k}}}
\end{array}\right),
$$

where $\varphi_{\mathbf{k}}$ is the angle between the atomic wave-vector $\mathbf{k}$ and the $x$-axis. The two-component spinors $g_{\mathbf{k}}^{ \pm}$are eigenfunctions of the chirality operator $\sigma_{\mathbf{k}}=\sigma \cdot \mathbf{k} / k$ representing a spin along the atomic motion, $\sigma_{\mathbf{k}} g_{\mathbf{k}}^{ \pm}= \pm g_{\mathbf{k}}^{ \pm}$. It should be emphasized that the chirality is here associated with the subspace of two dark states rather than with the spin in the usual sense.

The corresponding eigenenergies of the Hamiltonian (5) are isotropic, $\hbar \omega_{\mathbf{k}}^{ \pm} \equiv \hbar \omega_{k}^{ \pm}$, with

$$
\hbar \omega_{k}^{ \pm}=\frac{\hbar^{2}}{2 M}(k \pm \kappa)^{2}+\hbar \omega_{0}+V_{1}
$$

where $\omega_{0}=\hbar \kappa^{2} / 2 M$ is the recoil frequency. The relative dispersion $\omega_{k}^{ \pm} / \omega_{0}$ is plotted in Fig. 2 for $V_{1}=-\hbar \omega_{0}$. 


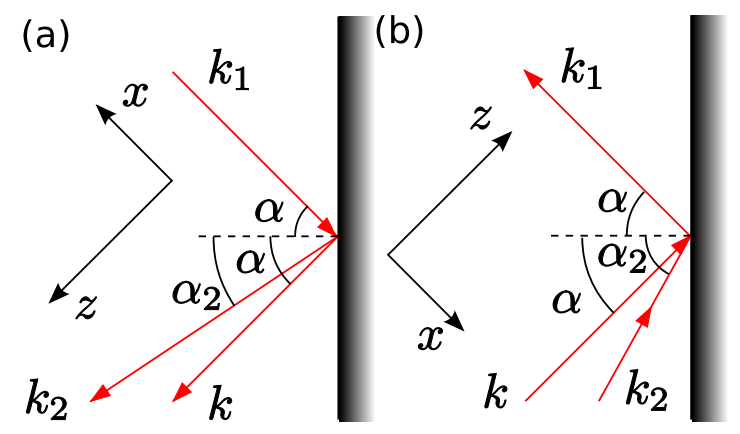

FIG. 3: (Color online) Reflection of atoms with negative chirality for $0<k<\kappa(\mathrm{a})$ and $\kappa<k<2 \kappa$ (b).

The upper (lower) dispersion branch is characterized by a positive (negative) chirality. For small wave-numbers $k \ll \kappa$ the dispersion is linear, $\omega_{k}^{ \pm} \sim \pm k$, so the atoms behave like ultra-relativistic Dirac fermions [16] similar to electrons in graphene [17]. For larger wave-numbers each $k=k_{1}<2 \kappa$ has a counterpart, $k_{2}=2 \kappa-k$, characterized by the same eigen-frequency $\hbar \omega_{k_{2}}^{-}=\hbar \omega_{k_{1}}^{-}$and opposite slope in the lower dispersion branch [23]. As shown below, this leads to unusual reflection properties.

Double and negative reflection of atoms To prepare an incident atom in the lower dispersion branch we suggest the following procedure. Initially the first two lasers are on, the third laser is off, and the atom is in the internal state $|3\rangle$ coinciding (up to a phase) with the second dark state, $\left|D_{2}\right\rangle=|3\rangle \exp \left(-i \kappa^{\prime} z\right)$. The atomic center of mass motion is initially characterized by a wave-vector $\mathbf{k}_{\mathrm{in}}=k_{\mathrm{in}} \mathbf{e}_{z}$ 24], giving the full state vector $|\Phi\rangle=\left|D_{2}\right\rangle \exp (i k z)$, where $k=k_{\text {in }}+\kappa^{\prime}$. Subsequently the laser 3 is switched on slowly, so that the atom remains in the dark state $\left|D_{2}\right\rangle$. Yet the duration of the switching-on should be short enough to avoid any substantial atomic motion at this stage. Since $\mathbf{k}=k \mathbf{e}_{z}$, the spinor $g_{\mathbf{k}}^{-}$represents the dark state $\left|D_{2}\right\rangle$. Hence one arrives at the atomic state-vector $|\Phi\rangle$ corresponding to the negative-chirality solution $\Psi_{\mathbf{k}}^{-}$, as required. The atoms prepared in this way will propagate along the $z$ axis for $k>\kappa$ or opposite to it for $k<\kappa$.

The atoms are impinging on an infinitely high potential barrier at an angle of incidence $\alpha$. We shall take $k<2 \kappa$, so that both reflected waves $\Psi_{\mathbf{k}_{1}}^{-}$and $\Psi_{\mathbf{k}_{2}}^{-}$remain in the lower dispersion branch with wave-numbers $k_{1}=k$ and $k_{2}=2 \kappa-k$. Fig. 3b shows the case of an incident wave with $\kappa<k<2 \kappa$. Here the group velocity $v_{k}^{-}=\partial \omega_{k}^{-} / \partial k$ is positive, so the wave-vectors of the incident and second reflected waves point inwards to the surface, whereas the wave-vector of the first reflected wave points outwards from the surface. Since $v_{k}^{-}=v_{k_{1}}^{-}=-v_{k_{2}}^{-}$, this ensures the forward propagation of the incident wave and backward propagation of the reflected ones. Fig. 3a illustrates a situation where $0<k<\kappa$. Here the group velocity $v_{k}^{-}$ is negative and hence the wave-vectors are reversed.

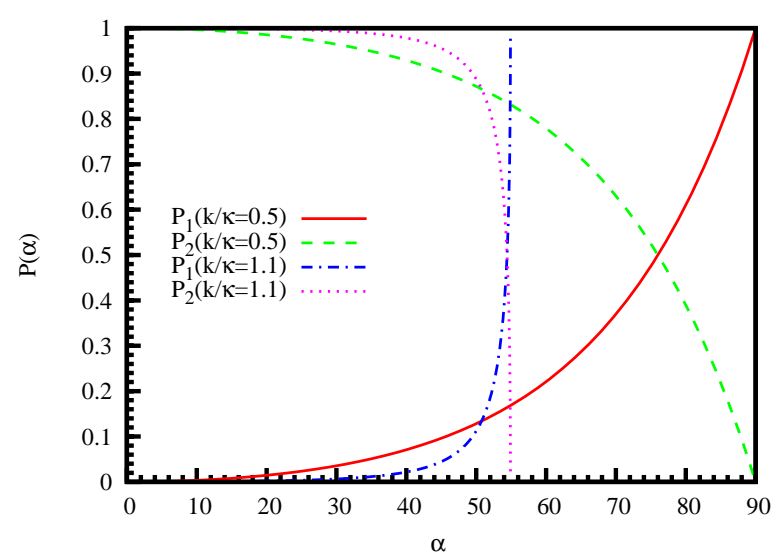

FIG. 4: (Color online) Reflection probabilities $P_{1}$ and $P_{2}$ for $k / \kappa=0.5$ and $k / \kappa=1.1$.

In front of the barrier the solution to the stationary Schrödinger equation $\left(H-\hbar \omega_{k}^{-}\right) \Psi=0$ is a linear superposition of the incident wave and two reflected waves:

$$
\Psi=\Psi_{\mathbf{k}}^{-}+r_{1} \Psi_{\mathbf{k}_{1}}^{-}+r_{2} \Psi_{\mathbf{k}_{2}}^{-} .
$$

The wave-vector is conserved along the reflection plane, $k_{\|}=k_{1 \|}=k_{2 \|}$, so the first wave exhibits an ordinary reflection with the reflection angle equal to the angle of incidence, $\alpha_{1}=\alpha$. The second wave is characterized by the opposite group velocity $v_{k_{2}}^{-}=-v_{k}^{-}=-v_{k_{1}}^{-}$, and hence it experiences a negative reflection at an angle

$$
\alpha_{2}=\arcsin \left[\sin (\alpha) k / k_{2}\right] .
$$

The reflection coefficients $r_{1}$ and $r_{2}$ are determined using Eqs. (6) and (8) together with the boundary condition at the potential barrier $\left.\Psi\right|_{\text {barrier }}=0$, giving

$$
r_{1}=\frac{e^{i \alpha}-e^{i \alpha_{2}}}{e^{-i \alpha}+e^{i \alpha_{2}}}, \quad r_{2}=-1-r_{1} .
$$

The corresponding reflection probabilities are

$$
P_{1}=\left|r_{1}\right|^{2}, \quad P_{2}=\frac{\cos \alpha_{2}}{\cos \alpha}\left|r_{2}\right|^{2},
$$

with $P_{1}+P_{2}=1$, where the weight factor $\cos \alpha_{2} / \cos \alpha$ appears when calculating the flow of atoms in and out of the surface for the incident and reflected waves. The probabilities $P_{1}$ and $P_{2}$ plotted in Figure 4 are seen to depend both on the angle of incidence $\alpha$ and also on the wave-number $k$. For small angles, $\alpha \ll 1$, there is predominantly a negative reflection to the second branch, $\left|P_{1}\right| \ll 1$ and $\left|P_{2}\right| \approx 1$. For large angles of incidence $(\alpha \rightarrow \pi / 2)$ and $0<k<\kappa$ we have mostly a specular reflection to the first branch, $\left|P_{2}\right| \ll 1$ and $\left|P_{1}\right| \approx 1$.

If $\kappa<k<2 \kappa$, the situation is more complex. In this case the second reflected wave becomes evanescent when the angle of incidence $\alpha$ exceeds a critical value given by 

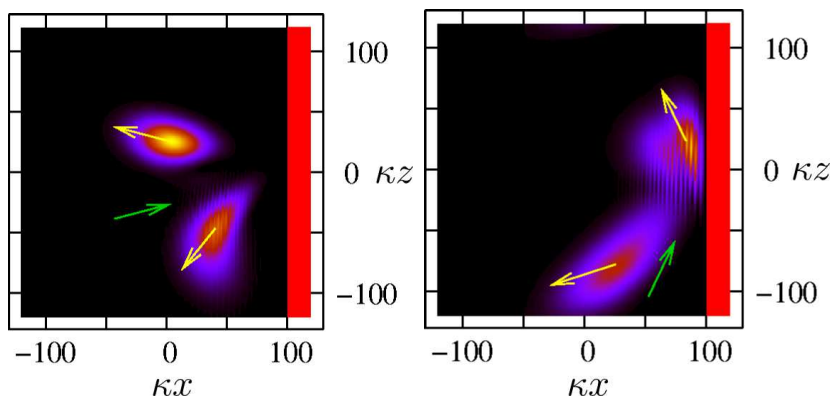

FIG. 5: (Color online) Reflection of an atomic wave-packet with a negative chirality for $\alpha=15^{\circ}, k=1.5 \kappa$ (left) and $\alpha=$ $65^{\circ}, k=0.5 \kappa$ (right). The incident wave-packet is taken to be Gaussian with momentum width $\Delta k=0.1 \kappa$. An additional arrow indicates the incident direction.

$\sin \alpha_{\text {crit }}=k_{2} / k$, i.e. for $k_{\|}=k_{2 \|}>k_{2}$. Consequently the out-of-plane projection of the wave-vector $\mathbf{k}_{2}$ becomes imaginary, $k_{2 \perp}=-i q$, with $q=\sqrt{k_{\|}^{2}-k_{2}^{2}}$. In the region where $x<0$ we can once again use Eq. (8) in which $\Psi_{\mathbf{k}_{2}}^{-}$ is now an evanescent wave. The boundary condition at the potential barrier gives the reflection coefficient

$$
r_{1}=\frac{e^{i \alpha} \sqrt{k_{\|}+q}-i \sqrt{k_{\|}-q}}{e^{-i \alpha} \sqrt{k_{\|}+q}+i \sqrt{k_{\|}-q}},
$$

with $\left|r_{1}\right|=1$. Thus there is a total reflection to the first mode at an angle $\alpha_{1}=\alpha$ accompanied by a phase shift, with the second reflected wave being evanescent. The phenomenon resembles the total internal reflection of optical waves at an interface with an optically thinner medium. In our situation, however, the evanescent wave is the reflected wave rather than the refracted one.

Our plane-wave analysis may be easily extended to the case of wavepacket reflection. Similar results may be found if the momentum width of the wavepacket $\Delta k$ is sufficiently small with respect to $\kappa$. Fig. 5 displays the double and negative reflection of atomic wavepackets from an atomic mirror, for an incident wavepacket $\Psi(\mathbf{r})=g_{\overline{\mathbf{k}}}^{-} e^{i \overline{\mathbf{k}} \cdot \mathbf{r}} f(r)$, with $f(r)$ a Gaussian, and $\overline{\mathbf{k}}$ the central wavenumber. The propagation direction and population of each of the reflected wave-packets are in good agreement with the analytical plane-wave results (9)(11). Similar results are also found for more realistic Gaussian or evanescent atomic mirrors [1, 2], as long as the potential barrier is sufficiently high compared to the incident kinetic energy. Lower barriers would lead to partial reflection, transmission and tunneling, whose physics will be the subject of further investigations.

Summarizing, the reflection of atoms under a nonAbelian gauge potential presents unusual features. In particular, one can have a double reflection comprising a specular and a non-specular one. Remarkably, the latter wave shows negative reflection due to the special properties of the dispersion law, and becomes evanescent for sufficiently large incident angles. Atom mirrors are a key tool in atom optics. Hence the anomalous reflection properties may be of crucial importance for the design of non Abelian atom optics elements, e.g. atom interferometers which exploit the non-Abelian Aharanov-Bohm effect.

This work was supported by the Royal Society of Edinburgh, the UK Engineering and Physical Sciences Research Council, the Deutsche Forschungsgemeinschaft (SFB407, SPP1116), and the European Graduate College on Interference and Quantum Applications.

[1] V. Balykin et al, Phys. Rev. Lett. 60, 2137 (1988).

[2] T. M. Roach et al, Phys. Rev. Lett. 75, 629 (1995).

[3] L. D. Barron, Molecular Light Scattering and Optical Activity (Cambridge University Press, Cambridge, England, 2004).

[4] A. Ghosh and P. Fischer, Phys. Rev. Lett. 97, 173002 (2006).

[5] A. F. Andreev, Sov. Phys. JETP 19, 1228 (1964).

[6] C. W. J. Beenakker, Phys. Rev. Lett. 97, 067007 (2006).

[7] D. Jaksch and P. Zoller, New J. Phys. 5, 56 (2003).

[8] E. J. Mueller, Phys. Rev. A 70, 041603(R) (2004).

[9] K. Osterloh, M. Baig, L. Santos, P. Zoller, and M. Lewenstein, Phys. Rev. Lett. 95, 010403 (2005).

[10] G. Juzeliūnas and P. Öhberg, Phys. Rev. Lett. 93, 033602 (2004); G. Juzeliūnas, P. Öhberg, J. Ruseckas, and A. Klein, Phys. Rev. A 71, 053614 (2005).

[11] J. Ruseckas, G. Juzeliūnas, P. Öhberg, and M. Fleischhauer, Phys. Rev. Lett. 95, 010404 (2005).

[12] T. D. Stanescu, C. Zhang, and V. Galitski, Phys. Rev. Lett. 99, 110403 (2007).

[13] J. Y. Vaishnav and C W. Clark, Phys. Rev. Lett. 100, 153002 (2008).

[14] I. I. Satija, D. C. Dakin, and C. W. Clark, Phys. Rev. Lett. 97, 216401 (2006).

[15] A. Jacob, P. Öhberg, G. Juzeliūnas and L. Santos, Appl. Phys. B 89, 439 (2007).

[16] G. Juzeliūnas, J. Ruseckas, M. Lindberg, L. Santos, and P. Öhberg, Phys. Rev. A 77, 011802(R) (2008).

[17] K. S. Novoselov et al, Nature 438, 197 (2005); A. K. Geim and K. S. Novoselov, Nature Materials 6, 183 (2007).

[18] R. G. Unanyan, M. Fleischhauer, B. W. Shore, and K. Bergmann, Opt. Commun. 155, 144 (1998).

[19] R. G. Unanyan, B. W. Shore, and K. Bergmann, Phys. Rev. A 59, 2910 (1999).

[20] M. V. Berry, Proc. R. Soc. A 392, 45 (1984).

[21] F. Wilczek and A. Zee, Phys. Rev. Lett. 52, 2111 (1984).

[22] C. A. Mead, Rev. Mod. Phys. 64, 51 (1992).

[23] A similar kind of dispersion is featured for optical waves in a medium with a single chiral resonance considered by J. B. Pendry, Science 306, 1353 (2004), as one can see comparing our figure 2 with the figure 2c by Pendry.

[24] The cold atoms can be set in motion using various techniques, e.g. by means of a two-photon scattering which induces a recoil momentum $\hbar \mathbf{k}_{\text {in }}=\hbar \mathbf{k}_{2 \text { phot }}$ to the atoms, where $\mathbf{k}_{2 \text { phot }}$ is the wave-vector of the two-photon mismatch. See e.g. L. Deng et al., Nature 398, 218 (1999). 